\title{
Cost-Benefit Analysis of a System with Server Failure and Replacement of the Unit at Different Failure Modes Subject to Inspection
}

\author{
S.C.Malik \\ Dept. of Statistics \\ M.D.University \\ Rohtak-124001(India)
}

\author{
A.K.Dhankar \\ Dept. of Statistics \\ M.D. University \\ Rohtak-124001(India)
}

\author{
Ashish Kumar \\ Dept. of Statistics \\ M.D.University \\ Rohtak-124001(India)
}

\begin{abstract}
This paper deals with a reliability model developed for a single-unit system which may fail completely either directly from normal mode or via partial failure. There is a single server who inspects the unit at its partial and complete failure to examine the feasibility of repair. If repair of the unit at these stages is not feasible, it is replaced immediately by new one. The system remains operative with partially failed unit. The server is subjected to failure while conducting inspection and repair of the unit. Treatment is given to the server upon failure. The repair of the unit and treatment given to the server are considered as perfect. The failure time of the unit and server follow negative exponential while the distributions of inspection and repair times of the unit as well as the distribution of treatment time of the server are taken as arbitrary.

To carry out cost-benefit analysis, the expressions for several measures of system effectiveness are derived using semiMarkov process and regenerative point technique. The graphical behavior of MTSF, availability and profit has been shown with respect to treatment rate of the server keeping fixed values of other parameters.
\end{abstract}

\section{General Terms}

Reliability and Server Failure

\section{Keywords}

Single-unit System, Server Failure, Replacement of the Unit, Treatment, Inspection and Cost-Benefit Analysis.

\section{INTRODUCTION}

In reliability theory, it is proved that performance and reliability of repairable systems can be improved by the method of redundancy. But there are many systems in which a unit cannot be kept as spare due to its high cost. Therefore, reliability models of single-unit systems with different failure modes have also been probed by the authors including Malik and Bansal [1], Malik [2] and Pawar and Malik[3] keeping in view of their practical utility and common man's affordability. In most of these papers, it is assumed that repair facility neither fails nor deteriorates. In fact, this assumption becomes unrealistic whenever server facility meets with an accident due to one reason or the other. And, in such situation, server may be given some treatment for curability. Recently, Dhankar and Malik [4] have studied reliability models of a single-unit system under different failure modes and server failure during inspection and repair. In these models, it is assumed that repair of the unit at its complete failure is always possible. Again, this assumption seems to be impracticable because repair of a unit at any stage of its failure more or less depends on the type of faults - repairable or non repairable. This can be revealed by inspection. And, if inspection reveals that unit is not repairable, it can be replaced by new one in order to avoid the unnecessary expenses on repair.

While considering the above facts and observations, this paper is devoted to the analysis of a reliability model developed for a single-unit system which may fail completely either directly from normal mode or via partial failure. The system remains operative with the partially failed unit. There is a single server who visits the system immediately to do repair activities at different failure modes. The server inspects the unit at its partial and complete failure to see the feasibility of repair. If repair of the unit at these failure modes is not feasible, it is replaced by new one. The server is subjected to failure while performing jobs. Treatment is given to the server upon failure for his curability. The repair of the unit and treatment given to the server are considered as perfect.

All random variables are uncorrelated and statistically independent. The switch devices are fault free. The failure time of the unit and server follow negative exponential whereas the distributions of inspection and repair time of the unit as well as the distribution of treatment time of the server are taken as arbitrary with different probability density functions. To carry out cost-benefit analysis, the expressions for transition probabilities and mean sojourn times, mean time to system failure (MTSF), availability, Busy period analysis, expected number of inspections by the server, expected number of treatments given to the server, expected number of visits by the server, expected number of replacements of the unit at its both failure modes and profit function in steady state are derived using semi-Markov process and regenerative point techniques. The graphical study of the results has also been made for a particular case.

\section{NOTATIONS}

E : Set of regenerative states.

O : The unit is operative and in normal mode.

SG : The server is good.

$\lambda / \lambda_{1} / \lambda_{2}$ : Constant failure rate of the unit from normal mode to complete failure / normal mode to partial failure / 
partial failure mode to complete failure.

$\mathrm{PU}_{\mathrm{i}} / \mathrm{PUr} / \mathrm{PW}_{\mathrm{i}} / \mathrm{PW}_{\mathrm{r}}$ :The unit is partially failed and under inspection /under repair / waiting for inspection / waiting for repair

$\mathrm{FU}_{\mathrm{i}} / \mathrm{FUr} / \mathrm{FW}_{\mathrm{i}} / \mathrm{FW}_{\mathrm{r}}$ : The unit is completely failed and under inspection/ under repair/waiting for inspection / waiting for repair.

$\mathrm{SFU}_{\mathrm{t}} / \mathrm{SFU}_{\mathrm{T}}$ : The server is failed and under treatment / under Treatment continuously from previous state.

$\mu \quad$ : Constant failure rate of the server.

$\mathrm{p} / \mathrm{q}$ : Probability that repair of the unit at partial failure is

$\mathrm{m} / \mathrm{n}$ : Probability that repair of the unit at complete failure is not feasible / feasible.

$\mathrm{g}(\mathrm{t}) / \mathrm{G}(\mathrm{t}) ; \mathrm{g}_{1}(\mathrm{t}) / \mathrm{G}_{1}(\mathrm{t})$ :pdf / cdf of repair time of the completely failed unit and partially failed unit.

$\mathrm{f}(\mathrm{t}) / \mathrm{F}(\mathrm{t})$ : pdf / cdf of treatment time of the server.

$\mathrm{h}_{1}(\mathrm{t}) / \mathrm{H}_{1}(\mathrm{t})$ :pdf / cdf of inspection time of the unit at partial failure.

$\mathrm{k}(\mathrm{t}) / \mathrm{K}(\mathrm{t})$ : pdf / cdf of inspection time of the unit at complete failure.

$\mathrm{q}_{\mathrm{ij}}(\mathrm{t}) / \mathrm{Q}_{\mathrm{ij}}(\mathrm{t})$ :pdf andedf of direct transition time from a regenerative state $\mathrm{i}$ to a regenerative state $\mathrm{j}$ without visiting any other regenerative state.

$\mathrm{q}_{\mathrm{iij} . \mathrm{k}}(\mathrm{t}) / \mathrm{Q}_{\mathrm{ij} . \mathrm{k}}(\mathrm{t})$ : pdf and cdf of first passage time from aregenerative state $i$ to a regenerative state $j$ or to $a$ failed state $\mathrm{j}$ visiting state $\mathrm{k}$ once in $(0, \mathrm{t}]$.

$\mathrm{M}_{\mathrm{i}}(\mathrm{t})$ : Probability that the system is up initially in state $\mathrm{S}_{\mathrm{i}} \in \mathrm{E}$ is up at time $t$ without visiting to any other regenerative state.

$\mathrm{W}_{\mathrm{i}}(\mathrm{t})$ : Probability that the server is busy in the state $\mathrm{S}_{\mathrm{i}}$ upto time ' $t$ 'without making any transition to any other regenerative state or returning to the same state via one or more non-regenerative states.

$\mathrm{m}_{\mathrm{ij}} \quad$ : Contribution to mean sojourn time $\left(\mu_{\mathrm{i}}\right)$ in state $\mathrm{S}_{\mathrm{i}}$

$$
\begin{aligned}
& \text { when system transit } \\
& \text { directly to state } S_{j} \text { so that } \\
& \mu_{i}=\sum_{j} m_{i j} \\
& \mathrm{~m}_{\mathrm{ij}}=\int t d Q_{i j}(t)=-q_{i j}^{*}(0)
\end{aligned}
$$

(s) / ( ) :Symbol for Stieltjes convolution / Laplace convolution

\footnotetext{
$\sim / *$ : Symbol for Laplace Stieltjes

Transform(LST)/Laplace Transform (LT).
}

$/($ desh):Symbol for derivative of the function.

The transition states $\mathrm{S}_{0}, \mathrm{~S}_{1}, \mathrm{~S}_{2}, \mathrm{~S}_{3}, \mathrm{~S}_{4}, \mathrm{~S}_{5}, \mathrm{~S}_{6}, \mathrm{~S}_{8}, \mathrm{~S}_{9}$ are regenerative and state $S_{7}$ is non regenerative as shown in figure 1 .

\section{RELIABILITY INDICES}

\subsection{Transition Probabilities and Mean} Sojourn Times

Simple probabilistic considerations yield the following expressions for the non-zero elements

$\mathrm{p}_{\mathrm{ij}}=\mathrm{Q}_{\mathrm{ij}}(\infty)=\int_{0}^{\infty} q_{\mathrm{ij}}(\mathrm{t}) \mathrm{dt}$ as we get

$\mathrm{p}_{06}=\frac{\lambda 1}{\lambda+\lambda 1}, \mathrm{p}_{08}=\frac{\lambda}{\lambda+\lambda 1}, \quad \mathrm{p}_{10}=\mathrm{g}_{1}^{8}\left(\mu+\lambda_{2}\right)$,

$\left.\mathrm{p}_{18}=\frac{\lambda_{2}}{\text { Holtreasible } / \text { feasible. }}\left(1-\mathrm{g}_{2}\right)\right)$,

$p_{13}=\frac{\mu}{\mu+\lambda 2}\left(1-g_{1}^{*}\left(\mu+\lambda_{2}\right)\right), \quad p_{20}=g^{*}(\mu)$

$, p_{23}=1-g^{*}(\mu), p_{31}=p_{56}=f^{*}\left(\lambda_{2}\right)$

$\mathrm{p}_{38.7}=\mathrm{p}_{58.7}=\left\{1-\mathrm{f}^{*}\left(\lambda_{2}\right)\right\} \mathrm{f}^{*}(0), \mathrm{p}_{42}=\mathrm{p}_{78}=\mathrm{p}_{98}=\mathrm{f}^{*}(0)$

$\mathrm{p}_{61}=\mathrm{q} h_{1}^{*}\left(\mu+\lambda_{2}\right), \mathrm{p}_{65}=\frac{\mu}{\left(\mu+\lambda_{2}\right)}\left[1-h_{1}^{*}\left(\mu+\lambda_{2}\right)\right]$

, $\mathrm{p}_{68}=\frac{\lambda_{2}}{\left(\mu+\lambda_{2}\right)}\left[1-h_{1}^{*}\left(\mu+\lambda_{2}\right)\right]$

$\mathrm{p}_{80}=\mathrm{mk}^{*}(\mu), \mathrm{p}_{82}=\mathrm{mk}^{*}(\mu), \mathrm{p}_{89}=1-\mathrm{k}^{*}(\mu)$

It can be easily verified that

$\mathrm{p}_{06}+\mathrm{p}_{08}=\mathrm{p}_{10}+\mathrm{p}_{13}+\mathrm{p}_{18}=\mathrm{p}_{20}+\mathrm{p}_{24}=\mathrm{p}_{31}+\mathrm{p}_{38.7}=\mathrm{p}_{42}$

$=\mathrm{p}_{56}+\mathrm{p}_{58.7}=\mathrm{p}_{60}+\mathrm{p}_{61}+\mathrm{p}_{65}+\mathrm{p}_{68}=\mathrm{p}_{80}+\mathrm{p}_{82}+\mathrm{p}_{89}=\mathrm{p}_{98}=1$

\section{Mean Sojourn times are}

$\mu_{0}=\mathrm{m}_{02}+\mathrm{m}_{09}=\int_{0}^{\infty} P(\mathrm{~T}>\mathrm{t}) \mathrm{dt}=\frac{1}{\lambda+\lambda 1}, \mu_{1}=\mathrm{m}_{10+}$

$\mathrm{m}_{13}+\mathrm{m}_{18}=\frac{\left(1-\mathrm{g}_{1}^{8}\left(\mu+\lambda_{2}\right)\right)}{\left(\mu+\lambda_{2}\right)}$

$\mu_{2}=m_{20+} m_{24}=\frac{1-g *(\mu)}{\mu}, \quad \mu_{3}=m_{31}+m_{37}$

$=\frac{\left[1-f^{*}\left(\lambda_{2}\right)\right]}{\lambda_{2}}$

$\mu_{3}^{z}=\mathrm{m}_{31+} \mathrm{m}_{38.7}=\mu_{5}^{z}=\mathrm{m}_{56+} \mathrm{m}_{58.7}=$

$\frac{\left[1-f^{*}\left(\lambda_{2}\right)\right]\left[1-\lambda_{2} f^{*}(0)\right]}{\lambda_{2}}$

$\mu_{4}=m_{42}=\mu_{9}=m_{98}=-f^{*^{I}}(0), \mu_{6}=m_{60}+$

$m_{61}+m_{65}+m_{68}=\frac{\left[1-h_{1}^{8}\left(\mu+\lambda_{2}\right)\right]}{\left(\mu+\lambda_{2}\right)}, \quad \mu_{8}=m_{80}+m_{82+} m_{89}$

$=\frac{\left[1-k^{8}(\mu)\right]}{\mu}$ 


\subsection{Reliability and Mean Time to System Failure (MTSF)}

Let $\Phi_{\mathrm{i}}(\mathrm{t})$ be the cdf of the first passage time fron regenerative

state $\mathrm{i}$ to a failed state regarding the failed state as absorbing state. We have the following recursive relations for $\Phi_{\mathrm{i}}(\mathrm{t})$ :

$$
\begin{aligned}
& \Phi_{0}(\mathrm{t})=\mathrm{Q}_{06}(\mathrm{t})(\mathrm{s}) \Phi_{6}(\mathrm{t})+\mathrm{Q}_{08}(\mathrm{t}) \\
& \Phi_{1}(\mathrm{t})=\mathrm{Q}_{10}(\mathrm{t})(\mathrm{s}) \Phi_{0}(\mathrm{t})+\mathrm{Q}_{13}(\mathrm{t})(\mathrm{s}) \Phi_{3}(\mathrm{t})+\mathrm{Q}_{18}(\mathrm{t}) \\
& \Phi_{3}(\mathrm{t})=\mathrm{Q}_{31}(\mathrm{t})(\mathrm{s}) \Phi_{1}(\mathrm{t})+\mathrm{Q}_{37}(\mathrm{t}) \\
& \Phi_{5}(\mathrm{t})=\mathrm{Q}_{56}(\mathrm{t})(\mathrm{s}) \Phi_{6}(\mathrm{t})+\mathrm{Q}_{57}(\mathrm{t}) \\
& \Phi_{6}(\mathrm{t})=\mathrm{Q}_{60}(\mathrm{t})(\mathrm{s}) \Phi_{0}(\mathrm{t})+\mathrm{Q}_{61}(\mathrm{t})(\mathrm{s}) \Phi_{1}(\mathrm{t})+ \\
& \mathrm{Q}_{65}(\mathrm{t})(\mathrm{s}) \Phi_{5}(\mathrm{t})+\mathrm{Q}_{68}(\mathrm{t})
\end{aligned}
$$

Taking LST of above relations (1) to obtain $\widetilde{\Phi}_{0}(s)$ Using

this, we have

$\mathrm{R}^{*}(\mathrm{~s})=\frac{1-\Phi_{0}(s)}{s}$

The reliability $\mathrm{R}(\mathrm{t})$ of the system model can be obtained by taking Laplace inverse transform of (2). The mean time to system failure is given by

$$
\mathrm{MTSF}=\lim _{\Omega \rightarrow 0} \mathrm{R}^{*}(\mathrm{~s})
$$

MTSF

$$
=\frac{\left(1-p_{13} p_{31}\right)\left\{?_{0}\left(1-p_{56} p_{65}\right)+?_{5} p_{06} p_{65}+?_{6} p_{06}\right\}}{\left(1-p_{13} p_{31}\right)\left(1-p_{56} p_{65}-p_{06} p_{60}\right)-p_{06} p_{10} p_{61}}
$$

\subsection{Steady State Availability}

Let $\mathrm{A}_{\mathrm{i}}(\mathrm{t})$ be the probability that the system is in upstate a instant

$t$ given that the system entered regenerative state $i$ at $t=0$. The recursive relations for $\mathrm{A}_{\mathrm{i}}(\mathrm{t})$ are as follows:

$$
\begin{aligned}
& A_{0}(t)=M_{0}(t)+q_{06}(t) \odot A_{6}(t)+q_{08}(t) \odot A_{8}(t) \\
& A_{1}(t)=M_{1}(t)+q_{10}(t) \odot A_{0}(t)+q_{13}(t) \odot A_{3}(t)+q_{18}(t) \odot A_{8}(t) \\
& A_{2}(t)=q_{20}(t) \odot A_{0}(t)+q_{24}(t) \odot A_{4}(t), A_{3}(t)=M_{3}(t)+ \\
& q_{31}(t) \odot A_{1}(t)+q_{38.7}(t) \odot A_{8}(t) \\
& A_{4}(t)=q_{42}(t) \odot A_{2}(t), A_{5}(t)=M_{5}(t)+q_{56}(t) \odot A_{6}(t) \\
& +q_{58.7}(t) \odot A_{8}(t) \\
& A_{6}(t)=M_{6}(t)+q_{60}(t) \odot A_{0}(t)+q_{61}(t) \odot A_{1}(t)+q_{65}(t) \\
& \odot A_{5}(t)+q_{68}(t) \odot A_{8}(t) \\
& A_{8}(t)=q_{80}(t) \odot A_{0}(t)+q_{82}(t) \odot A_{2}(t)+q_{89}(t) \odot A_{9}(t) \\
& A_{9}(t)=q_{98}(t) \odot A_{8}(t) \\
& \text { where }
\end{aligned}
$$

$$
\begin{aligned}
& \mathrm{M}_{0}(\mathrm{t})=\mathrm{e}^{-(\lambda+\lambda 1) \mathrm{t}}, \quad \mathrm{M}_{1}(\mathrm{t})=\mathrm{e}^{-(\mu+\lambda 2) t} \bar{G}_{1}(\mathrm{t}), \mathrm{M}_{3}(\mathrm{t})= \\
& \mathrm{M}_{5}(\mathrm{t})=\mathrm{e}^{-\lambda 2 \mathrm{t}} \bar{F}(\mathrm{t}), \quad \mathrm{M}_{6}(\mathrm{t})=\mathrm{e}^{-(\mu+\lambda 2) t} \bar{H}_{1}(\mathrm{t})
\end{aligned}
$$

Now taking L.T. of relations (4) and obtain the value of $\mathrm{A}_{0}{ }^{*}(\mathrm{~s})$. By using this, the steady state availability is given by

$$
\begin{aligned}
& \mathrm{A}_{10}=\lim _{z \rightarrow 0} \mathrm{sA}_{0}{ }^{*}(\mathrm{~s})=\mathrm{N}_{11} / \mathrm{D}_{11}, \text { where } \\
& \mathrm{N}_{11}=p_{20}\left(1-p_{89}\right)\left[\left(1-p_{13} p_{31}\right)\{\right. \\
& \left.\left(1-p_{56} p_{65}\right) ?_{0}+p_{06}\left(?_{5} p_{65}+?_{6}\right)\right\} \\
& +p_{06} p_{61}\left(?_{1}+\quad ?_{3} p_{13}\right) \\
& \mathrm{D}_{11}= \\
& p_{20}\left(1-p_{89}\right)\left[\left(1-p_{13} p_{31}\right)\right. \\
& \left\{?_{0}\left(1-p_{56} p_{65}\right)+?_{5}^{\prime} p_{06} p_{65}+?_{6} p_{06}\right\} \\
& \left.+\quad p_{06} p_{61}\left(?_{1}+?_{3}^{\prime} p_{13}\right)\right]+ \\
& \left\{\left(1-p_{13} p_{31}\right)\left(1-p_{56} p_{65}-p_{06} p_{60}\right)\right. \\
& \left.-\quad p_{06} p_{10} p_{61}\right\}\left\{p_{82}\left(?_{2}+?_{4} p_{24}\right)\right. \\
& \left.+p_{20}\left(?_{8}+?_{9} p_{89}\right)\right\}
\end{aligned}
$$

\subsection{Busy Period Analysis for the Server}

Let $\mathrm{B}_{\mathrm{i}}(\mathrm{t})$ be the probability that the server is busy at instant $t$ given that the system entered regenerative state $i$ at $t=0$. The recursive relation for $\mathrm{B}_{\mathrm{i}}(\mathrm{t})$ are as follows:

$$
\begin{aligned}
& \mathrm{B}_{0}(\mathrm{t})=\mathrm{q}_{06}(\mathrm{t}) \odot \mathrm{B}_{6}(\mathrm{t})+\mathrm{q}_{08}(\mathrm{t}) \odot \mathrm{B}_{8}(\mathrm{t}) \\
& \mathrm{B}_{1}(\mathrm{t})=\mathrm{W}_{1}(\mathrm{t})+\mathrm{q}_{10}(\mathrm{t}) \odot \mathrm{B}_{0}(\mathrm{t})+\mathrm{q}_{13}(\mathrm{t}) \odot \mathrm{B}_{3}(\mathrm{t})+\mathrm{q}_{18}(\mathrm{t}) \odot \mathrm{B}_{8}(\mathrm{t}) \\
& \mathrm{B}_{2}(\mathrm{t})=\mathrm{W}_{2}(\mathrm{t})+\mathrm{q}_{20}(\mathrm{t}) \odot \mathrm{B}_{0}(\mathrm{t})+\mathrm{q}_{24}(\mathrm{t}) \odot \mathrm{B}_{4}(\mathrm{t}) \\
& \mathrm{B}_{3}(\mathrm{t})=\mathrm{q}_{31}(\mathrm{t}) \odot \mathrm{B}_{1}(\mathrm{t})+\mathrm{q}_{38.7}(\mathrm{t}) \odot \mathrm{B}_{8}(\mathrm{t}), \mathrm{B}_{4}(\mathrm{t})=\mathrm{q}_{42}(\mathrm{t}) \odot \mathrm{B}_{2}(\mathrm{t}) \\
& \mathrm{B}_{5}(\mathrm{t})=\mathrm{q}_{56}(\mathrm{t}) \odot \mathrm{B}_{6}(\mathrm{t})+\mathrm{q}_{58.7}(\mathrm{t}) \odot \mathrm{B}_{8}(\mathrm{t}) \\
& \mathrm{B}_{6}(\mathrm{t})=\mathrm{W}_{6}(\mathrm{t})+\mathrm{q}_{60}(\mathrm{t}) \odot \mathrm{B}_{0}(\mathrm{t})+\mathrm{q}_{61}(\mathrm{t}) \odot \mathrm{B}_{1}(\mathrm{t})+\mathrm{q}_{65}(\mathrm{t}) \odot \mathrm{B}_{5}(\mathrm{t}) \\
& +\mathrm{q}_{68}(\mathrm{t}) \odot \mathrm{B}_{8}(\mathrm{t}) \\
& \mathrm{B}_{8}(\mathrm{t})=\mathrm{W}_{8}(\mathrm{t})+\mathrm{q}_{80}(\mathrm{t}) \odot \mathrm{B}_{0}(\mathrm{t})+\mathrm{q}_{82}(\mathrm{t}) \odot \mathrm{B}_{2}(\mathrm{t})+\mathrm{q}_{89}(\mathrm{t}) \odot \mathrm{B}_{9}(\mathrm{t}) \\
& \mathrm{B}_{9}(\mathrm{t})=\mathrm{q}_{98}(\mathrm{t}) \odot \mathrm{B}_{8}(\mathrm{t})
\end{aligned}
$$

where,

$\mathrm{W}_{1}(\mathrm{t})=\mathrm{e}^{-(\mu+\lambda 2) t} \bar{G}_{1}(\mathrm{t}), \mathrm{W}_{2}(\mathrm{t})=\mathrm{e}^{-\mu \mathrm{t}} \bar{G}(\mathrm{t}), \mathrm{W}_{6}(\mathrm{t})=\mathrm{e}^{-(\mu+\lambda 2) \mathrm{t}}$ $\bar{H}_{1}(t), \mathrm{W}_{2}(\mathrm{t})=\mathrm{e}^{-\mu \mathrm{t}} \bar{K}(t)$

Now taking L.T. of relations (6) and obtain the value of $\mathrm{B}_{0}{ }^{*}(\mathrm{~s})$ and by using this, the time for which server is busy in steady state is given by

$\mathrm{B}_{10}=\lim _{\boldsymbol{\Sigma} \rightarrow 0} \mathrm{sB}_{0}{ }^{*}(\mathrm{~s})=\mathrm{N}_{12} / \mathrm{D}_{11}$,

where 
$\mathrm{N}_{12}=p_{06} p_{20}\left(1-p_{89}\right)\left\{?_{6}\left(1-p_{13} p_{31}\right)+?_{1} p_{61}\right\}$

$+\left[p_{06} p_{61}\left(p_{13} p_{38.7}+p_{18}\right)+\right.$

$\left(1-p_{13} p_{31}\right)$

$\left.\left\{p_{06} p_{68}+p_{08}\left(1-p_{56} p_{65}\right)+p_{06} p_{58.7} p_{65}\right\}\right]$

$\left(?_{8} p_{20}+?_{2} p_{82}\right)$

and $\mathrm{D}_{11}$ is already defined

\subsection{Expected Number of Inspections by the Server}

Let $I_{i}(t)$ be the expected number of inspections by the server in $(0, t]$ given that the system entered regenerative state $i$ at $t=0$ The recursive relations for $\mathrm{I}_{\mathrm{i}}(\mathrm{t})$ are as follows:

$\mathrm{I}_{0}(\mathrm{t})=\mathrm{Q}_{06}(\mathrm{t})(\mathrm{s})\left[1+\mathrm{I}_{6}(\mathrm{t})\right]+\mathrm{Q}_{08}(\mathrm{t})(\mathrm{s})\left[1+\mathrm{I}_{8}(\mathrm{t})\right]$

$\mathrm{I}_{1}(\mathrm{t})=\mathrm{Q}_{10}(\mathrm{t})(\mathrm{s}) \mathrm{I}_{0}(\mathrm{t})+\mathrm{Q}_{13}(\mathrm{t})(\mathrm{s}) \mathrm{I}_{3}(\mathrm{t})+\mathrm{Q}_{18}(\mathrm{t})(\mathrm{s})\left[1+\mathrm{I}_{8}(\mathrm{t})\right]$

$\mathrm{I}_{2}(\mathrm{t})=\mathrm{Q}_{20}(\mathrm{t})(\mathrm{s}) \mathrm{I}_{0}(\mathrm{t})+\mathrm{Q}_{24}(\mathrm{t})(\mathrm{s}) \mathrm{I}_{4}(\mathrm{t}), \mathrm{I}_{3}(\mathrm{t})=\mathrm{Q}_{31}(\mathrm{t})(\mathrm{s}) \mathrm{I}_{1}(\mathrm{t})$

$+\mathrm{Q}_{38.7}(\mathrm{t})(\mathrm{s})\left[1+\mathrm{I}_{8}(\mathrm{t})\right]$

$\mathrm{I}_{4}(\mathrm{t})=\mathrm{Q}_{42}(\mathrm{t})(\mathrm{s}) \mathrm{I}_{2}(\mathrm{t}), \mathrm{I}_{5}(\mathrm{t})=\mathrm{Q}_{56}(\mathrm{t})(\mathrm{s})\left[1+\mathrm{I}_{6}(\mathrm{t})\right]+\mathrm{Q}_{58.7}(\mathrm{t})$

(s) $\left[1+\mathrm{I}_{8}(\mathrm{t})\right]$

$\mathrm{I}_{6}(\mathrm{t})=\mathrm{Q}_{60}(\mathrm{t})(\mathrm{s}) \mathrm{I}_{0}(\mathrm{t})+\mathrm{Q}_{61}(\mathrm{t})(\mathrm{s}) \mathrm{I}_{1}(\mathrm{t})+\mathrm{Q}_{65}(\mathrm{t})(\mathrm{s}) \mathrm{I}_{5}(\mathrm{t})+$ $\mathrm{Q}_{68}(\mathrm{t})(\mathrm{s}) \mathrm{I}_{8}(\mathrm{t})$

$\mathrm{I}_{8}(\mathrm{t})=\mathrm{Q}_{80}(\mathrm{t})(\mathrm{s}) \mathrm{I}_{0}(\mathrm{t})+\mathrm{Q}_{82}(\mathrm{t})(\mathrm{s}) \mathrm{I}_{2}(\mathrm{t})+\mathrm{Q}_{89}(\mathrm{t})(\mathrm{s}) \mathrm{I}_{9}(\mathrm{t})$ $\mathrm{I}_{9}(\mathrm{t})=\mathrm{Q}_{98}(\mathrm{t})(\mathrm{s})\left[1+\mathrm{I}_{8}(\mathrm{t})\right]$

Now taking L.S.T. of relation (7) and solving for $\widetilde{\mathrm{I}}_{0}(s)$. By using this, the expected numbers of inspections carried out by the server in steady state are given by

$\mathrm{I}_{10}=\lim _{s \rightarrow 0} \tilde{\mathrm{I}}_{0}(s)=\mathrm{N}_{13} / \mathrm{D}_{11}$

Where

$\mathrm{N}_{13}=p_{20}\left(1-p_{13} p_{31}\right)$

$\left\{\left(1-p_{56} p_{65}-p_{06} p_{89}+p_{06}\left(p_{65}+p_{68} p_{89}\right)\right\}\right.$

$+p_{06} p_{20} p_{61}\left(p_{13} p_{38.7}+p_{18}\right)$

and, $D_{11}$ is already defined.

\subsection{Expected Number of Treatments Given To the Server}

Let $T_{i}(t)$ be the expected number of Treatments given to the server in $(0, t]$ such that the system entered regenerative state $\mathrm{i}$ at $\mathrm{t}=0$. The recursive relations for $\mathrm{T}_{\mathrm{i}}(\mathrm{t})$ are as follows:

$$
\begin{aligned}
& \mathrm{T}_{0}(\mathrm{t})=\mathrm{Q}_{06}(\mathrm{t})(\mathrm{s}) \mathrm{T}_{6}(\mathrm{t})+\mathrm{Q}_{08}(\mathrm{t})(\mathrm{s}) \mathrm{T}_{8}(\mathrm{t}) \\
& \mathrm{T}_{1}(\mathrm{t})=\mathrm{Q}_{10}(\mathrm{t})(\mathrm{s}) \mathrm{T}_{0}(\mathrm{t})+\mathrm{Q}_{18}(\mathrm{t})(\mathrm{s}) \mathrm{T}_{8}(\mathrm{t})+\mathrm{Q}_{13}(\mathrm{t})(\mathrm{s})\left[1+\mathrm{T}_{3}(\mathrm{t})\right] \\
& \mathrm{T}_{2}(\mathrm{t})=\mathrm{Q}_{20}(\mathrm{t})(\mathrm{s}) \mathrm{T}_{0}(\mathrm{t})+\mathrm{Q}_{24}(\mathrm{t})(\mathrm{s})\left[1+\mathrm{T}_{4}(\mathrm{t})\right] \\
& \mathrm{T}_{3}(\mathrm{t})=\mathrm{Q}_{31}(\mathrm{t})(\mathrm{s}) \mathrm{T}_{1}(\mathrm{t})+\mathrm{Q}_{38.7}(\mathrm{t})(\mathrm{s}) \mathrm{T}_{8}(\mathrm{t}) \\
& \mathrm{T}_{4}(\mathrm{t})=\mathrm{Q}_{42}(\mathrm{t})(\mathrm{s}) \mathrm{T}_{2}(\mathrm{t}), \mathrm{T}_{5}(\mathrm{t})=\mathrm{Q}_{56}(\mathrm{t})(\mathrm{s}) \mathrm{T}_{6}(\mathrm{t})+\mathrm{Q}_{58.7}(\mathrm{t})(\mathrm{s}) \mathrm{T}_{8}(\mathrm{t}) \\
& \mathrm{T}_{6}(\mathrm{t})=\mathrm{Q}_{60}(\mathrm{t})(\mathrm{s}) \mathrm{T}_{0}(\mathrm{t})+\mathrm{Q}_{61}(\mathrm{t})(\mathrm{s}) \mathrm{T}_{1}(\mathrm{t})+\mathrm{Q}_{65}(\mathrm{t})(\mathrm{s})\left[1+\mathrm{T}_{5}(\mathrm{t})\right] \\
& +\mathrm{Q}_{68}(\mathrm{t})(\mathrm{s}) \mathrm{T}_{8}(\mathrm{t}) \\
& \mathrm{T}_{8}(\mathrm{t})=\mathrm{Q}_{80}(\mathrm{t})(\mathrm{s}) \mathrm{T}_{0}(\mathrm{t})+\mathrm{Q}_{82}(\mathrm{t})(\mathrm{s}) \mathrm{T}_{2}(\mathrm{t})+\mathrm{Q}_{89}(\mathrm{t})(\mathrm{s})\left[1+\mathrm{T}_{9}(\mathrm{t})\right], \ldots(8) \\
& \mathrm{T}_{9}(\mathrm{t})=\mathrm{Q}_{98}(\mathrm{t})(\mathrm{s}) \mathrm{T}_{8}(\mathrm{t})
\end{aligned}
$$

Now taking L.S.T. of relations (8) and solving for $\widetilde{\mathrm{T}}_{0}(s)$ and by using this, the expected number of the treatments given to server are given by

$$
\begin{aligned}
& \mathrm{T}_{10}=\lim _{s \rightarrow 0} \mathrm{~s} \widetilde{\mathrm{T}}_{0}(s)=\mathrm{N}_{14} / \mathrm{D}_{11}, \text { where } \\
& \mathrm{N}_{14}=\left(p_{89} p_{20}+p_{24} p_{82}\right) \\
& \left\{\left(1-p_{13} p_{31}\right)\left(1-p_{56} p_{65}-p_{06} p_{60}\right)-p_{06} p_{10} p_{61}\right\} \\
& +p_{06} p_{20}\left(1-p_{89}\right)\left\{p_{65}\left(1-p_{13} p_{31}\right)+p_{13} p_{61}\right\}
\end{aligned}
$$

and $\mathrm{D}_{11}$ is already defined.

\subsection{Expected Number of Treatments Given to the Server}

Let $\mathrm{N}_{\mathrm{i}}(\mathrm{t})$ be the expected number of visits by the server in $(0, \mathrm{t}$ given that the system entered regenerative state $i$ at $t=0$.Th recursive

relations for $\mathrm{N}_{\mathrm{i}}(\mathrm{t})$ are as follows:

$$
\begin{aligned}
& N_{0}(t)=Q_{06}(t)(s)\left[1+N_{6}(t)\right]+Q_{08}(t)(s)\left[1+N_{8}(t)\right] \\
& N_{1}(t)=Q_{10}(t)(s) N_{0}(t)+Q_{13}(t)(s) N_{3}(t)+Q_{18}(t)(s) N_{8}(t) \ldots(9) \\
& N_{2}(t)=Q_{20}(t)(s) N_{0}(t)+Q_{24}(t)(s) N_{4}(t) \\
& N_{3}(t)=Q_{31}(t)(s)\left[1+N_{1}(t)\right]+Q_{38.7}(t)(s)\left[1+N_{8}(t)\right] \\
& N_{4}(t)=Q_{42}(t)(s)\left[1+N_{2}(t)\right], N_{5}(t)=Q_{56}(t)(s)\left[1+N_{6}(t)\right]+ \\
& Q_{58.7}(t)(s)\left[1+N_{8}(t)\right] \\
& N_{6}(t)=Q_{60}(t)(s) N_{0}(t)+Q_{61}(t)(s) N_{1}(t)+Q_{65}(t)(s) N_{5}(t)+ \\
& Q_{68}(t)(s) N_{8}(t) \\
& N_{8}(t)=Q_{80}(t)(s) N_{0}(t)+Q_{82}(t)(s) N_{2}(t)+Q_{89}(t)(s) N_{9}(t) \\
& N_{9}(t)=Q_{98}(t)(s)\left[1+N_{8}(t)\right]
\end{aligned}
$$


Now taking L.S.T. of relations (9) and solving for $\widetilde{\mathrm{N}}_{0}(s)$, the expected number of visits by the server are given by

$$
\begin{aligned}
& \mathrm{N}_{10}=\lim _{\boldsymbol{s} \rightarrow 0} \mathrm{~s} \widetilde{\mathrm{N}}_{0}(s)=\mathrm{N}_{15} / \mathrm{D}_{11} \text {, where } \\
& \mathrm{N}_{15}=\left(p_{89} p_{20}+p_{82} p_{24}\right) \\
& \left\{\left(1-p_{13} p_{31}\right)\left(1-p_{56} p_{65}-p_{06} p_{60}\right)\right. \\
& \left.-p_{06} p_{10} p_{61}\right\} \\
& +\left(1-p_{89}\right) p_{20}\left\{( 1 - p _ { 1 3 } p _ { 3 1 } ) \left(1-p_{56} p_{65}\right.\right. \\
& \left.+p_{06} p_{65}\right) \\
& \left.+p_{06} p_{13} p_{61}\right\}
\end{aligned}
$$

\subsection{Expected Number of Replacements of The Unit}

Let $R_{i}(t)$ be the expected number of replacements of the unit in $(0, t]$ given that the system entered regenerative state $\mathrm{i}$ at $\mathrm{t}=0$. The recursive relations for $\mathrm{R}_{\mathrm{i}}(\mathrm{t})$ are as follows:

$$
\begin{aligned}
& \mathrm{R}_{0}(\mathrm{t})=\mathrm{Q}_{06}(\mathrm{t})(\mathrm{s}) \mathrm{R}_{6}(\mathrm{t})+\mathrm{Q}_{08}(\mathrm{t})(\mathrm{s}) \mathrm{R}_{8}(\mathrm{t}) \\
& \mathrm{R}_{1}(\mathrm{t})=\mathrm{Q}_{10}(\mathrm{t})(\mathrm{s}) \mathrm{R}_{0}(\mathrm{t})+\mathrm{Q}_{13}(\mathrm{t})(\mathrm{s}) \mathrm{R}_{3}(\mathrm{t})+\mathrm{Q}_{18}(\mathrm{t})(\mathrm{s}) \mathrm{R}_{8}(\mathrm{t}) \\
& \mathrm{R}_{2}(\mathrm{t})=\mathrm{Q}_{20}(\mathrm{t})(\mathrm{s}) \mathrm{R}_{0}(\mathrm{t})+\mathrm{Q}_{24}(\mathrm{t})(\mathrm{s}) \mathrm{R}_{4}(\mathrm{t}) \\
& \mathrm{R}_{3}(\mathrm{t})=\mathrm{Q}_{31}(\mathrm{t})(\mathrm{s}) \mathrm{R}_{1}(\mathrm{t})+\mathrm{Q}_{38.7}(\mathrm{t})(\mathrm{s}) \mathrm{R}_{8}(\mathrm{t}) \quad \ldots(10) \\
& \mathrm{R}_{4}(\mathrm{t})=\mathrm{Q}_{42}(\mathrm{t})(\mathrm{s}) \mathrm{R}_{2}(\mathrm{t}), \quad \mathrm{R}_{5}(\mathrm{t})=\mathrm{Q}_{56}(\mathrm{t})(\mathrm{s}) \mathrm{R}_{6}(\mathrm{t})+\mathrm{Q}_{58.7}(\mathrm{t})(\mathrm{s} \\
& \mathrm{R}_{8}(\mathrm{t}) \\
& \mathrm{R}_{6}(\mathrm{t})=\mathrm{Q}_{60}(\mathrm{t})(\mathrm{s})\left[1+\mathrm{R}_{0}(\mathrm{t})\right]+\mathrm{Q}_{61}(\mathrm{t})(\mathrm{s}) \mathrm{R}_{1}(\mathrm{t})+\mathrm{Q}_{65}(\mathrm{t})(\mathrm{s}) \mathrm{R}_{5}(\mathrm{t}) \\
& +\mathrm{Q}_{68}(\mathrm{t})(\mathrm{s}) \mathrm{R}_{8}(\mathrm{t}) \\
& \mathrm{R}_{8}(\mathrm{t})=\mathrm{Q}_{80}(\mathrm{t})(\mathrm{s})\left[1+\mathrm{R}_{0}(\mathrm{t})\right]+\mathrm{Q}_{82}(\mathrm{t})(\mathrm{s}) \mathrm{R}_{2}(\mathrm{t})+\mathrm{Q}_{89}(\mathrm{t})(\mathrm{s}) \mathrm{R}_{9}(\mathrm{t}) \\
& \mathrm{R}_{9}(\mathrm{t})=\mathrm{Q}_{98}(\mathrm{t})(\mathrm{s}) \mathrm{R}_{8}(\mathrm{t})
\end{aligned}
$$

Now taking L.S.T. of relations (10) and solving for $\widetilde{R}_{0}(S)$, the expected number of visits by the server are given by

$$
\begin{aligned}
& \mathrm{R}_{10}=\lim _{s \rightarrow 0} \mathrm{~s} \tilde{R}_{0}(S)=\mathrm{N}_{16} / \mathrm{D}_{11}, \text { where } \\
& \mathrm{N}_{16}=p_{20}\left(1-p_{13} p_{31}\right) \\
& \left\{p_{80}\left(1-p_{56} p_{65}\right)+p_{06} p_{60} p_{82}\right\}- \\
& p_{06} p_{10} p_{20} p_{61} p_{80} \text { and } \mathrm{D}_{11} \text { is already defined }
\end{aligned}
$$

\subsection{Cost-Benefit Analysis}

Profit incurred to the system model in steady state is given by $\mathrm{P}=\mathrm{K}_{0} \mathrm{~A}_{10}-\mathrm{K}_{1} \mathrm{~B}_{10}-\mathrm{K}_{2} \mathrm{I}_{10}-\mathrm{K}_{3} \mathrm{~T}_{10}-\mathrm{K}_{4} \mathrm{~N}_{10}-\mathrm{K}_{5} \mathrm{R}_{10}$ where

$\mathrm{K}_{0}=$ Revenue per unit up time of the system.

$\mathrm{K}_{1}=$ Cost per unit time for which server is busy.

$\mathrm{K}_{2}=$ Cost per unit time inspection by the server.

$\mathrm{K}_{3}=$ Cost per unit time treatment given to the server.

$\mathrm{K}_{4}=$ Cost per unit visit by the server.

$\mathrm{K}_{5}=$ Cost per unit time replacement of the unit.

\section{CONCLUSION}

In the present study, results for a particular case $\mathrm{g}(\mathrm{t})=\alpha \mathrm{e}^{-\alpha . t}, \mathrm{~g}_{1}(\mathrm{t})=\alpha_{1} \mathrm{e}^{-\alpha .1 \mathrm{t}}, \mathrm{f}(\mathrm{t})=\beta \mathrm{e}^{-\beta \mathrm{t}}, \mathrm{h}_{1}(\mathrm{t})=\gamma_{1} \mathrm{e}^{-\gamma 1 \mathrm{t}}, \quad \mathrm{k}(\mathrm{t})$ $=\delta \mathrm{e}^{-\delta \mathrm{t}}$ are obtained. The mean time to system to system failure (MTSF) goes on increasing with the increase of treatment rate $(\beta)$ of the server, repair rate $\left(\alpha_{1}\right)$ and inspection rate $\left(\gamma_{1}\right)$ of the partially failed unit for fixed values of other parameters with $m=.6=p$ and $n=.4=q$ as shown in the table 1 . But the effect of repair rate $\left(\alpha_{1}\right)$ and inspection rate $\left(\gamma_{1}\right)$ of the completely failed unit is about negligible on MTSF. And, the value of MTSF decreases with the increase of different failure rates $\left(\lambda, \lambda_{1}, \lambda_{2}\right)$ and failure rate $(\mu)$ of the server. Again, it may be noted that MTSF become less by interchanging the values of $\mathrm{p}$ and $\mathrm{q}$. From tables 2 and 3, it is observed that availability and profit of the system model follow upward trend by increasing the treatment rate $(\beta)$, repair rates $\left(\alpha\right.$ and $\left.\alpha_{1}\right)$ and inspection rates $\left(\delta\right.$ and $\left.\gamma_{1}\right)$ for $\mathrm{m}=\mathrm{p}=.6$ and $\mathrm{n}=\mathrm{q}=.4$ with $\mathrm{K}_{0}=2000, \mathrm{~K}_{1}=500, \mathrm{~K}_{2}=\mathrm{K} 3=\mathrm{K}_{4}=200$ and $\mathrm{K}_{5}=100$. However, their values follow a decline trend with the increase of failures rates $\left(\lambda, \lambda_{1}, \lambda_{2}\right)$ and failure rate $(\mu)$ of the server. Further, it can be seen that the effect of repair rate $(\alpha)$ and direct failure rate $(\lambda)$ is much more on availability and profit of the system model as compared to other parameters. Also, the systems become less profitable by interchanging the values of $\mathrm{m}$ with $\mathrm{n}$ and $\mathrm{p}$ with $\mathrm{q}$.

Thus the study reveals that a single unit system with different failure modes and server failure can be made more economically beneficial in the following ways:

(i) By controlling the direct failure rate of the unit

(ii) By increasing inspection and repair rates of the completely failed unit.

(iii) By giving preference to the replacement of the failed unit by new one over repair in case server fails frequently.

\section{REFERENCES}

[1] Malik,S.C. and Bansal, R.K.( 2005) : Profit analysis of single-unit reliability models with repair at different failure modes. Proc. International Conference on Reliability and Safety Engineering, IIT Kharagpur (India), pp. 577-588.

[2] Malik,S.C. (2008) : Reliability modeling and profit analysis of a single-unit system with inspection by a server who appears and disappears randomly. Journal of Pure and Applied Mathematika Sciences, Vol.LXVII(12), pp. 135-146.

[3] Pawar, D. and Malik,S.C. (2010): Reliability and Economic Measures of a System with Inspection for Online Repair and No Repair Activity in Abnormal Weather. Bulletin of Pure and Applied Sciences, Vol. 29 $\mathrm{E}(2), \mathrm{pp} .355-368$.

[4] Dhankar,A.K. and Malik, S.C.(2011): Cost- Benefit Analysis of System Reliability Models with Server

[5] Failure During Inspection and Repair. International Journal of Statistics \& Analysis,Vol.1(3),pp. 265- 278. 


\section{STATE TRANSITION DIAGRAM}

Fig: 1

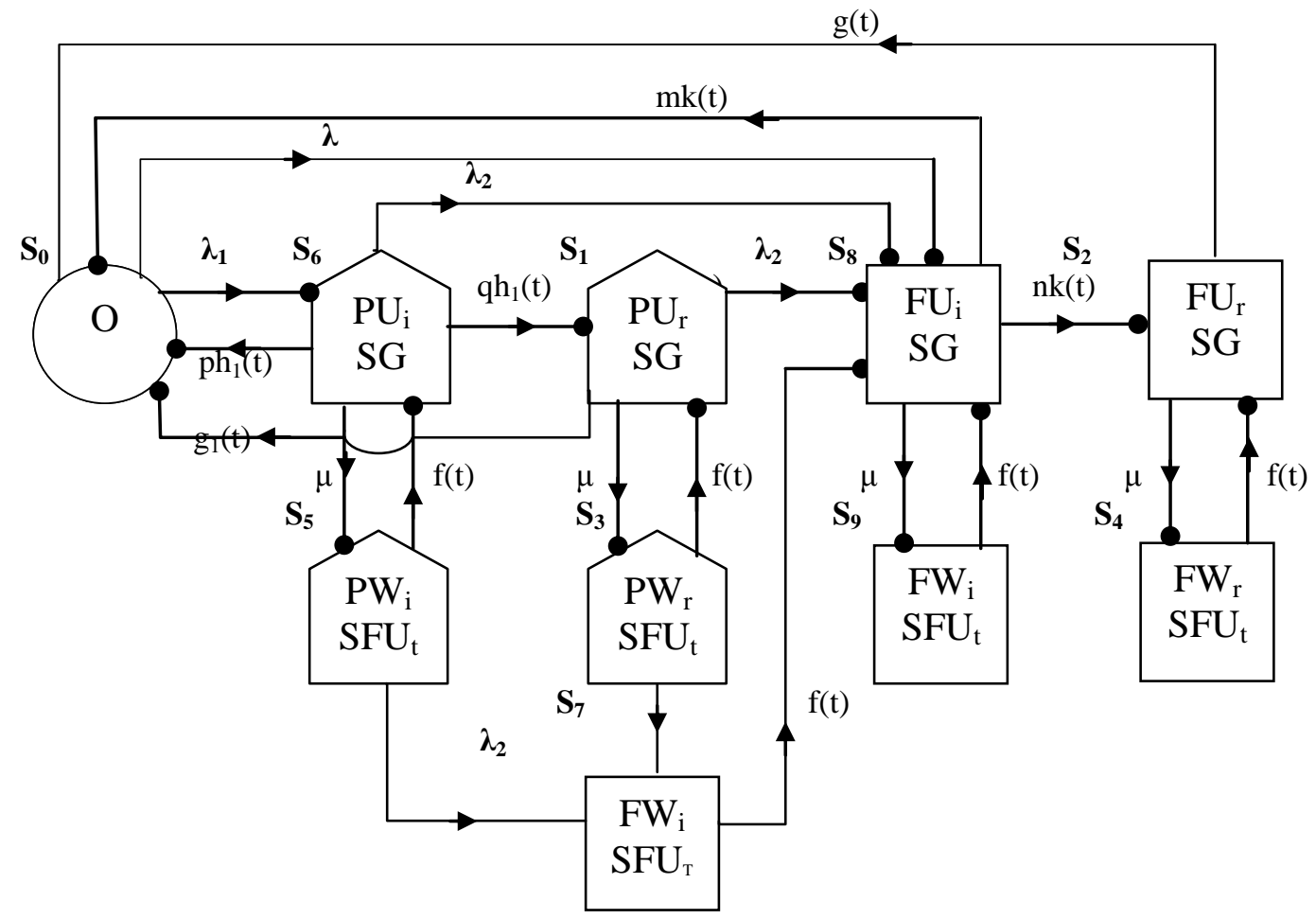

- : Transition point

$\square$ : Partially Failed

$\bigcirc$ : Up-State

Completely Failed 
Table 1: MTSF Vs. Treatment Rate

\begin{tabular}{|l|l|l|l|r|r|r|r|r|r|r|r|}
\hline$\beta$ & & $\alpha=1$ & $\alpha 1=2$ & $\delta=3.5$ & $\mu=0.1$ & $\gamma 1=4$ & $\lambda=0.3$ & $\lambda 1=0.5$ & $\lambda 2=0.7$ & $\mathrm{~m}=0.6, \mathrm{n}=0.4$ & $\mathrm{p}=0.6, \mathrm{q}=0.4$ \\
& $\alpha=1$ & $\alpha=1.1$ & $\alpha 1=2.1$ & $\delta=3.6$ & $\mu=0.2$ & $\gamma 1=4.1$ & $\lambda=0.4$ & $\lambda 1=0.6$ & $\lambda 2=0.8$ & $\mathrm{~m}=0.4, \mathrm{n}=0.6$ & $\mathrm{p}=0.4, \mathrm{q}=0.6$ \\
\hline 0.1 & 2.759 & 2.759 & 2.764 & 2.759 & 2.728 & 2.764 & 2.238 & 2.684 & 2.666 & 2.759 & 2.69 \\
0.2 & 2.763 & 2.763 & 2.768 & 2.763 & 2.735 & 2.767 & 2.24 & 2.687 & 2.67 & 2.763 & 2.694 \\
0.3 & 2.766 & 2.766 & 2.771 & 2.766 & 2.74 & 2.77 & 2.241 & 2.691 & 2.672 & 2.766 & 2.697 \\
0.4 & 2.768 & 2.768 & 2.773 & 2.768 & 2.745 & 2.773 & 2.242 & 2.693 & 2.675 & 2.768 & 2.699 \\
0.5 & 2.77 & 2.77 & 2.775 & 2.77 & 2.748 & 2.775 & 2.243 & 2.695 & 2.676 & 2.77 & 2.701 \\
0.6 & 2.772 & 2.772 & 2.777 & 2.772 & 2.752 & 2.777 & 2.244 & 2.697 & 2.678 & 2.772 & 2.703 \\
0.7 & 2.773 & 2.773 & 2.779 & 2.773 & 2.755 & 2.778 & 2.245 & 2.699 & 2.68 & 2.773 & 2.705 \\
\hline
\end{tabular}

Table 2: Availability Vs. Treatment Rate

\begin{tabular}{|l|l|l|r|r|r|r|r|r|r|r|r|}
\hline$\beta$ & & $\alpha=1$ & $\alpha 1=2$ & $\delta=3.5$ & $\mu=0.1$ & $\gamma 1=4$ & $\lambda=0.3$ & $\lambda 1=0.5$ & $\lambda 2=0.7$ & $\mathrm{~m}=0.6, \mathrm{n}=0.4$ & $\mathrm{p}=0.6, \mathrm{q}=0.4$ \\
& $\alpha=1$ & $\alpha=1.1$ & $\alpha 1=2.1$ & $\delta=3.6$ & $\mu=0.2$ & $\gamma 1=4.1$ & $\lambda=0.4$ & $\lambda 1=0.6$ & $\lambda 2=0.8$ & $\mathrm{~m}=0.4, \mathrm{n}=0.6$ & $\mathrm{p}=0.4, \mathrm{q}=0.6$ \\
\hline 0.1 & 0.618 & 0.628 & 0.619 & 0.62 & 0.503 & 0.619 & 0.577 & 0.605 & 0.612 & 0.567 & 0.606 \\
0.2 & 0.701 & 0.711 & 0.702 & 0.703 & 0.623 & 0.702 & 0.661 & 0.692 & 0.695 & 0.651 & 0.692 \\
0.3 & 0.734 & 0.743 & 0.734 & 0.736 & 0.676 & 0.734 & 0.694 & 0.726 & 0.727 & 0.685 & 0.726 \\
0.4 & 0.751 & 0.76 & 0.751 & 0.753 & 0.705 & 0.751 & 0.712 & 0.744 & 0.744 & 0.703 & 0.744 \\
0.5 & 0.761 & 0.771 & 0.762 & 0.763 & 0.724 & 0.762 & 0.723 & 0.755 & 0.755 & 0.714 & 0.756 \\
0.6 & 0.768 & 0.778 & 0.769 & 0.77 & 0.737 & 0.769 & 0.73 & 0.763 & 0.762 & 0.722 & 0.763 \\
0.7 & 0.774 & 0.783 & 0.774 & 0.776 & 0.746 & 0.774 & 0.736 & 0.768 & 0.768 & 0.727 & 0.768 \\
\hline
\end{tabular}

Table 3: Profit Vs. Treatment Rate

\begin{tabular}{|l|l|l|l|l|l|l|l|l|l|l|r|}
\hline$\beta$ & & $\alpha=1$ & $\alpha 1=2$ & $\delta=3.5$ & $\mu=0.1$ & $\gamma 1=4$ & $\lambda=0.3$ & $\lambda 1=0.5$ & $\lambda 2=0.7$ & $\mathrm{~m}=0.6, \mathrm{n}=0.4$ & $\mathrm{p}=0.6, \mathrm{q}=0.4$ \\
& $\alpha=1$ & $\alpha=1.1$ & $\alpha 1=2.1$ & $\delta=3.6$ & $\mu=0.2$ & $\gamma 1=4.1$ & $\lambda=0.4$ & $\lambda 1=0.6$ & $\lambda 2=0.8$ & $\mathrm{~m}=0.4, \mathrm{n}=0.6$ & $\mathrm{p}=0.4, \mathrm{q}=0.6$ \\
\hline 0.1 & 905.89 & 925.36 & 907.61 & 910.12 & 730.68 & 907.25 & 804.75 & 859.28 & 893.55 & 813.33 & 886.47 \\
0.2 & 1027.4 & 1046.9 & 1028.8 & 1031.6 & 903.36 & 1028.5 & 921.69 & 981.48 & 1014.2 & 933.96 & 1012 \\
0.3 & 1074.7 & 1094 & 1076.1 & 1078.9 & 979.5 & 1075.7 & 967.93 & 1029.5 & 1061.5 & 981.95 & 1061.3 \\
0.4 & 1099.7 & 1118.8 & 1100.9 & 1103.8 & 1021.9 & 1100.6 & 992.43 & 1054.8 & 1086.5 & 1007.5 & 1087.3 \\
0.5 & 1114.9 & 1133.9 & 1116.2 & 1119.1 & 1048.7 & 1115.8 & 1007.5 & 1070.3 & 1101.8 & 1023.3 & 1103.3 \\
0.6 & 1125.2 & 1144.1 & 1126.4 & 1129.3 & 1067.1 & 1126 & 1017.6 & 1080.7 & 1112.2 & 1033.9 & 1114 \\
0.7 & 1132.5 & 1151.3 & 1133.7 & 1136.6 & 1080.4 & 1133.3 & 1024.9 & 1088.2 & 1119.6 & 1041.5 & 1121.6 \\
\hline
\end{tabular}

